\title{
Reconfigurable Microwave Coupled Resonator Band-pass Filter and Diplexer
}

\author{
Lidiane da Silva Araujo \\ Department of Electronics and Systems \\ Federal University of Pernambuco \\ Recife, Brazil \\ lidiane_sac@hotmail.com \\ Ignacio Llamas-Garro \\ Centre Tecnològic de Telecomunicacions de Catalunya \\ Parc Mediterrani de la Tecnologia \\ Castelldefels, Spain \\ ignacio.1lamas@cttc.es
}

\author{
Antonio J. Belfort de Oliveira \\ Department of Electronics and Systems \\ Federal University of Pernambuco \\ Recife, Brazil \\ antonio.belfort@gmail.com \\ Fermín Mira \\ Centre Tecnològic de Telecomunicacions de Catalunya \\ Parc Mediterrani de la Tecnologia \\ Castelldefels, Spain \\ fermin.mira@cttc.cat
}

\author{
Michael J. Lancaster \\ Department of Electronic, Electrical and Systems Engineering \\ University of Birmingham \\ Birmingham, UK \\ m.j.lancaster@bham.ac.uk
}

\begin{abstract}
This paper presents the design, simulation and experimental results of a reconfigurable microstrip band-pass filter formed by two square open-loop resonators. A reconfigurable diplexer designed with four square spiral resonators is also proposed and preliminary simulation results are reported. The devices are designed to operate at $\mathrm{S}$-band, and the reconfiguration is achieved using PIN diodes.
\end{abstract}

Index Terms-reconfigurable filter, reconfigurable diplexer, PIN diodes, coupled resonators, coupling matrix

\section{INTRODUCTION}

Current telecommunication systems should handle multiple standards. For example, in mobile communications to allow several technologies to operate simultaneously, where reconfigurable devices can be used to select a given service. Microstrip components are compact, lightweight, easy to integrate with other devices, and low cost. They are able to provide solutions for mobile systems to reconfigure both the frequency of operation and the bandwidth for communication channel selection. There have been previous efforts on reducing the size of telecommunication hardware through reconfiguration these include filters based on straight and bent microstrip lines. The use of open-loop and spiral resonators leads to miniaturized circuits [1]-[4]. This paper focuses on the realization of a compact reconfigurable filter and a diplexer using open-loop and spiral resonators for further size reduction compared to other works [5]-[7].

This work is supported by the Spanish government under MICINN grant RTI2018-099841-B-I00. Part of this work has been supported by the Generalitat de Catalunya under grant 2017 SGR 891.
In section II of the paper, a two state, $0.043 \mathrm{~dB}$ ripple, Chebyshev two-pole microstrip filter is described. It operates at a frequency of $2.45 \mathrm{GHz}$ when the PIN diodes are in the ON state. When the PIN diodes are in OFF state, the filter has an operation frequency of $2.65 \mathrm{GHz}$. The design, simulation and experiential results are discussed. In section III the design and simulation of a reconfigurable microstrip diplexer is given. The design is based on square spiral resonators for high device miniaturization. The two bands center frequency of the diplexer are $2.36 \mathrm{GHz}$ and $2.63 \mathrm{GHz}$ in the didoes in $\mathrm{ON}$ state, and $2.43 \mathrm{GHz}$ and $2.74 \mathrm{GHz}$ in the diodes in OFF state. Both the reconfigurable filter and diplexer are designed on $1.5 \mathrm{~mm}$ thick, CuClad 217 dielectric substrate. BAR50-02V PIN didoes are used along with choke inductors having a self resonance at $2.6 \mathrm{GHz}$ and inductance of $62 \mathrm{nH}$. All the simulations are done with CST Microwave Studio [8].

\section{THE RECONFIGURABLE FILTER}

\section{A. Design}

The filter was originally designed without reconfiguration and following the standard coupling theory [9]. This original design is a 2-pole band-pass filter with a Chebyshev response operating at a central frequency of $2.455 \mathrm{GHz}$ with $3 \%$ of fractional bandwidth $(F B W)$, and a $-20 \mathrm{~dB}$ maximum reflection loss in the passband. These specifications result in the coupling coefficient $M_{12}=0.05$ and the external quality factors $Q_{e 1}=Q_{e 2}=22.16$. From these values the resonator dimensions and the spacing between them can be found for this non reconfigurable filter, giving an estimate of the structure of the filter. To add the reconfiguration, so there is a second 
operating band at $2.65 \mathrm{GHz}$, the resonator lengths need to be reduced. This is done by having a small gap in the microstrip line forming the resonator, and then placing a PIN diode to switch between the different lengths/frequencies. The external quality factors also need to be altered for the two states. This is done by decreasing the length of the feed line providing less overlap to the resonator and reducing the coupling, thus increasing the external $Q$. A photograph of the fabricated reconfigurable filter is shown in Fig. 1. The design uses four PIN diodes, one per resonator and one per feed line, all of them forward biased (ON state) or reverse biased (OFF state) at the same time to achieve the two device states of operation. The dimensions are optimized in CST microwave studio, and simulation results are provided in the next section with the experimental results.

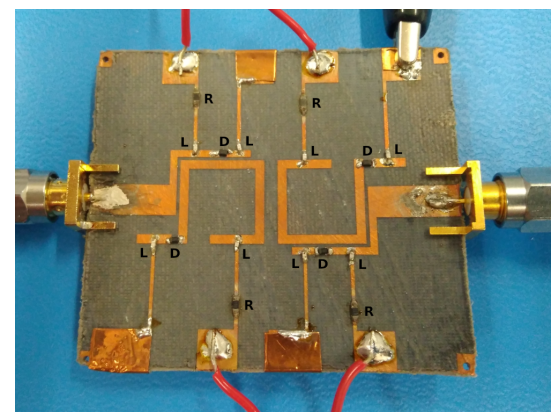

Fig. 1: Photograph of the 2-pole band-pass reconfigurable filter. D: diode, L: inductor, R: resistor.

\section{B. Experimental Results}

The reconfigurable filter has an ON state passband centered at $2.45 \mathrm{GHz}$, leading to the resonance frequency close to the original one (chosen for the original design). In the OFF state the filter operates at a higher resonance frequency of $2.65 \mathrm{GHz}$. Simulation and experimental results for the $\mathrm{S}$ parameters, $\left|S_{21}\right|_{\mathrm{dB}}$ and $\left|S_{11}\right|_{\mathrm{dB}}$, are shown in Fig. 2, considering the two states of the diodes.

It can be seen in Fig. 2 that although the return loss and the frequency accuracy are very good, the transmission loss is higher than expected. Losses can come from the narrow copper microstrip line, additional resistance in the inductors and pin diodes or radiation. Although all these have been included in the simulation, any deviation from the specified values entered the simulation will degrade the insertion loss. Work is underway on mounting the filter in a box to reduce radiation, as well as checking the PIN diodes and other components for these additional losses.

\section{THE RECONFIGURABLE DIPLEXER}

\section{A. Design}

Similarly to the filter design, the reconfigurable diplexer is firstly designed as an original version with no reconfigurability. This is a three port device with four square spiral resonators, coupled according to the topology shown in Fig. 3. The scheme is such that resonator 1 is connected to the input

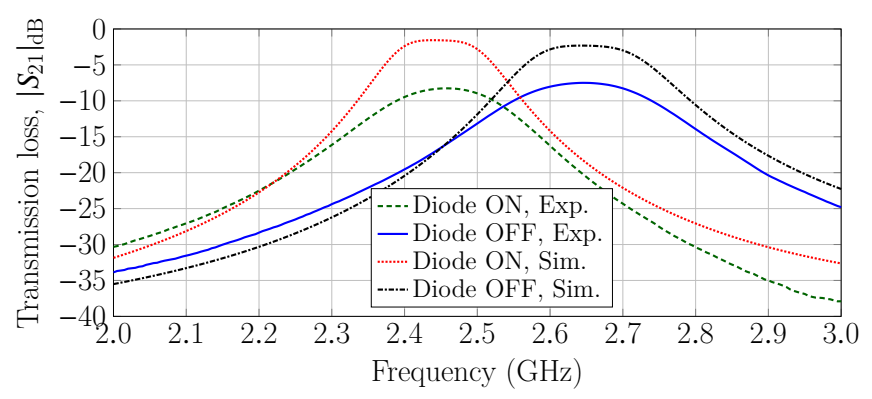

(a)

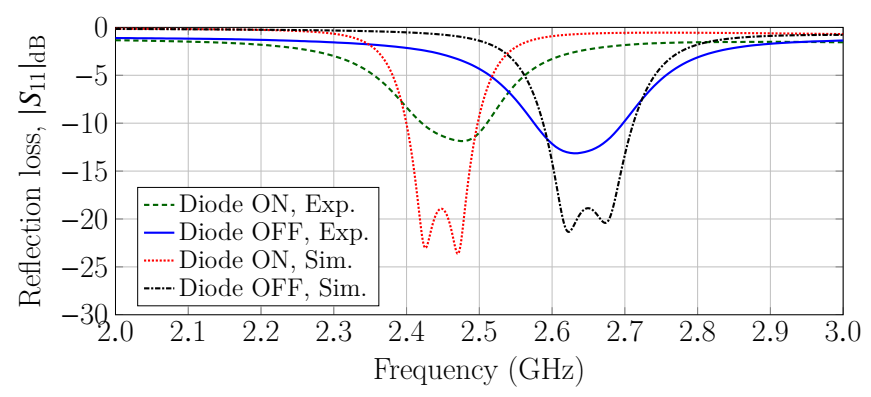

(b)

Fig. 2: Experimental and simulation results of the reconfigurable filter for the two device states. (a) Transmission loss $\left(20 \log \left|S_{21}\right|\right)$. (b) Reflection $\operatorname{loss}\left(20 \log \left|S_{11}\right|\right)$.

port (port 1) and operates at the same center frequency as resonator 2, which is the center frequency of the diplexer. Port 2 is connected to resonator 3 , and port 3 is connected to resonator 4 . With $F B W=14 \%$ and a center frequency of $2.5 \mathrm{GHz}$, the diplexer was designed following the coupling matrix theory for multiport networks in [10]. The coupling matrix $[m]$ was optimized for two $2^{\text {nd }}$-order Chebyshev responses with maximum reflection loss of $-16.4 \mathrm{~dB}$ (or $0.1 \mathrm{~dB}$ ripple) for each of the two bands of the diplexer. This led to $m_{12}=1.0676$ and $m_{23}=m_{24}=0.2738$, and values of the external quality factors of $q_{e 3}=q_{e 4}=5.8175$ and $q_{e 1}=q_{e 3} / 2$. The original diplexer was designed to operate in the bands around $f_{01}=2.34 \mathrm{GHz}$ (operating frequency of the resonator 4) and $f_{02}=2.65 \mathrm{GHz}$ (operating frequency of the resonator 3). The ideal frequency response obtained with the optimized matrix [m] is shown in Fig. 4.

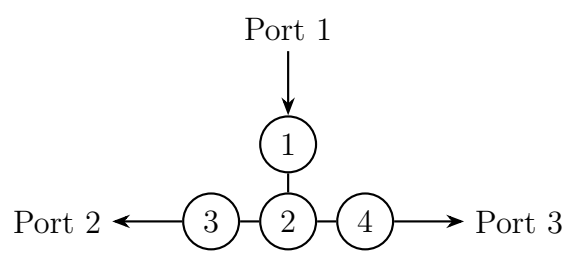

Fig. 3: The original diplexer topology.

The full reconfigurable diplexer design is shown in Fig. 5, with four square spiral resonators, bias lines, PIN diodes, choke inductors and resistors. The design is based on the filter described in section II, with the frequencies adjusted by 


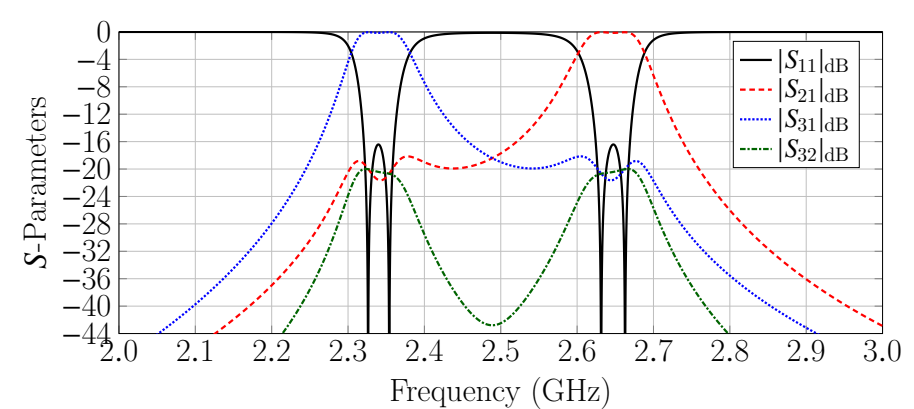

Fig. 4: Ideal responses plotted from the optimized coupling matrix of the diplexer.

varying the lengths of the resonator lines. The PIN diodes located near the end of the spiral resonators are biased using inductors and via holes to ground, avoiding undesired couplings and complex bias line structures. When the diodes are switched OFF, the length of each resonator is reduced and, as a result, the resonance frequencies increase and the diplexer continues to operate in distinct bands but with higher central frequencies. The resonators recover their original lenght when the diodes are switched ON.

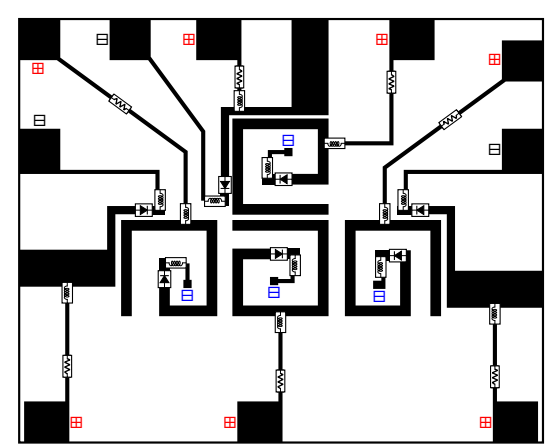

Fig. 5: Layout of the 4-resonator reconfigurable diplexer.

\section{B. Simulation Results}

The preliminary simulation results of the reconfigurable diplexer are shown in Fig. 6 including both operation states of the diplexer, according to diode $\mathrm{ON}$ and OFF states. The proposed design is compact and promising for the miniaturization of reconfigurable front-end transceiver channel selection.

\section{CONCLUSions}

A reconfigurable band-pass Chebyshev filter with two square open-loop coupled resonators is reported and includes simulation and experimental results. A good agreement in terms of frequency shift is obtained, however, the transmission loss in the passband is higher compared to simulations. A very compact reconfigurable diplexer topology has been introduced, including simulation results. The design uses spiral resonators to achieve device miniaturization.

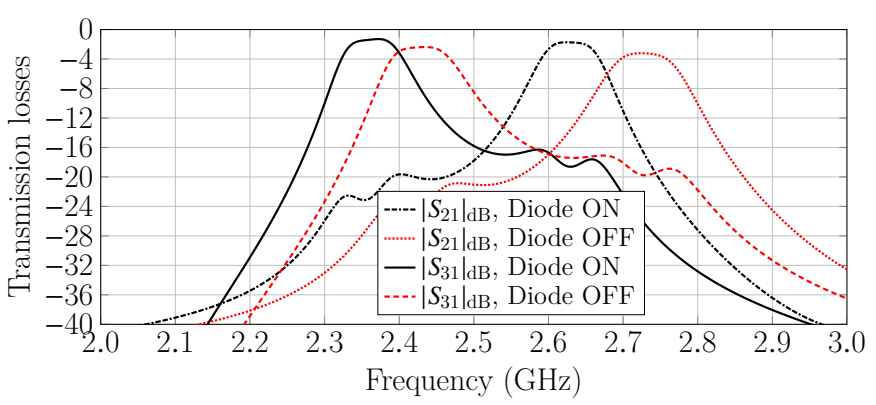

(a)

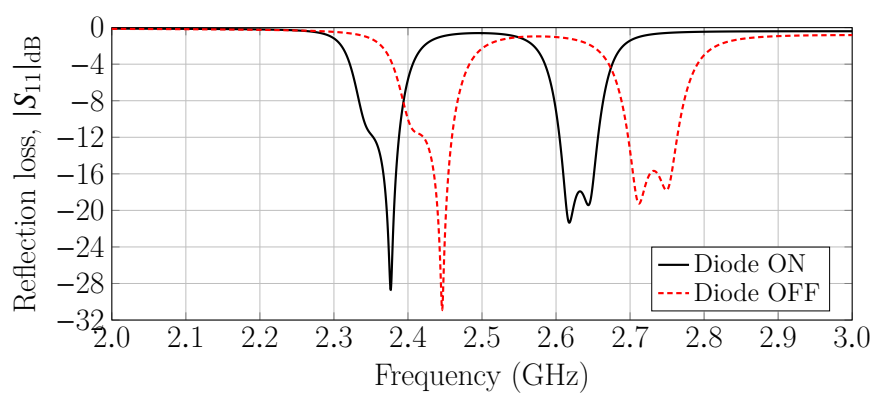

(b)

Fig. 6: Simulation results of the reconfigurable diplexer with layout shown in Fig. 5, for diodes ON and OFF states. (a) Transmission losses. (b) Reflection loss.

\section{REFERENCES}

[1] R. Wu and S. Amari, "New triangular microstrip loop resonators for bandpass dual-mode filter applications," IEEE MTT-S International Microwave Symposium Digest, 2005.

[2] L. S. Araujo, and A. J. B. de Oliveira, "A microstrip triangular spiral resonator for bandpass filter design,” SBMO/IEEE MTT-S International Microwave Optoelectronics Conference (IMOC2013), Rio de Janeiro, Brazil, 2013

[3] L. S. Araujo, and A. J. B. de Oliveira, "The square spiral resonator: Investigating its electromagnetic performance for filter design," SBMO/IEEE MTT-S International Microwave and Optoelectronics Conference (IMOC2015), Pernambuco, Brazil, 2015.

[4] X. Guan, et al, "Miniaturized high-temperature superconducting diplexer using common resonator and cross coupling structure," IEEE Transactions on Applied Superconductivity, Institute of Electrical and Electronics Engineers (IEEE), v. 27, n. 4, p. 1-4, jun 2017.

[5] Z. Brito-Brito, I. Llamas-Garro, and L. Pradell, "Selectivity Tuned Bandpass Filter," Electronics Letters, Vol. 45, No. 19, September 2009, pp. 984-985.

[6] Z. Brito-Brito, I. Llamas-Garro, and L. Pradell, "Precise Frequency and Bandwidth Control of Microstrip Switchable Bandstop Filters," Microwave and Optical Technology Letters, Wiley, Vol. 51, No. 11, November 2009, pp 2573-2578.

[7] Z. Brito-Brito, I. Llamas-Garro, G. Navarro-Muñoz, J. PerruisseauCarrier, and L. Pradell, "Switchable Bandpass Filter for WiFi-UMTS Reception Standards,' Electronics Letters, Vol. 46, No. 13, pp. 930-931, June 2010.

[8] Computer Simulation Technology (CST), Microwave Studio, 2017. Available at http:www.cst.com

[9] J. S. Hong and M. J. Lancaster, Microstrip filters for RF/Microwave Applications. Wiley, New York, USA, 2001.

[10] T. F. Skaik, "Synthesis of coupled resonator circuits with multiple outputs using coupling matrix optimization”. PhD thesis - School of Electronic, Electrical and Computer Engineering The University of Birmingham, March 2011. 